\title{
Physical Optics Characterization of a THz Time Domain System: UWB Leaky Lens Antenna vs. Austin Switch
}

\author{
N. Llombart ${ }^{1}$, A. Neto $^{2}$, P. H. Siegel ${ }^{3}$ \\ ${ }^{1}$ Optics Department, Universidad Complutense de Madrid, Spain \\ ${ }^{2}$ Telecom Dept. EEMCS, Technical University of Delft - Delft, The Netherlands. \\ ${ }^{3}$ California Institute of Technology, Pasadena, USA
}

\begin{abstract}
In this work we show that a THz time domain system based on photoconductive antennas can be improved by over one order of magnitude by using the Ultra-wideband (UWB) leaky lens antenna. The system has been analyzed by implementing a physical optics method to characterize radiated fields by a silicon lens.
\end{abstract}

\section{INTRODUCTION AND BACKGROUND}

$\mathrm{A}$ $\mathrm{N}$ innovation in the terahertz spectroscopy field (realized only within this decade) is the fast pulsed optical time domain spectrometer (THz TDS). This instrument uses femtosecond optical pulses to generate electrons in a photoconductive switch [1]. Most of such systems operate in frequency bandwidths of $1: 10$ and more. The system performances rely on the antenna response, both in terms of radiation efficiency and time domain pulse distortion [2]. Most of the TDS systems use an Austin switch consisting on a small dipole-like antenna. If this dipole is resonant, its radiated fields will vary significantly over of such large bandwidth introducing nulls in the spectrum, whereas if the dipole length is smaller than the resonance, it will generate a broad flat spectrum, but with a very low radiation efficiency due to the fact that the impedance will be practically imaginary.

Another important aspect with such TDS antennas is that they are coupled to an optical system (usually consisting of a couple of parabolic or elliptical mirrors) in order to perform the spectroscopic analysis. This means that the antenna needs to be directive. For such reason and to avoid the excitation of surface modes in the substrate, the Austin switch dipole is usually placed at focus of a silicon lens.

UWB antennas have been extensively study in the microwave regime, but they are usually dispersive, and not directive or inefficient. Recently, a revolutionary new UWB antenna has been proposed [3]. The antenna has already been demonstrated to work and preserve the pulse fidelity in the microwave regime, from 10 to $70 \mathrm{GHz}$ [4].

In this work, we study the use of such an antenna to improve the performances of standard THz TDS systems. This contribution consists on the analysis of the power spectrum response by using a standard physical optics method. The results are compared with a standard Austin Switch emitter, and show that the system could be improved by over 2 orders of magnitude.

\section{ANALYSIS METHOD}

When analyzing the TDS, most studies assumed the antenna as a point source for which the radiated electric field is directly proportional to the time-derivative of the current [5]. Some studies have included the impact of antenna length incorporating the Smith model and the silicon lens [6] and are able to reproduce the maximum and minimum found in the TD spectrum.

Here we want to study the impact of the antenna geometry on the TDS spectral properties in order to show the enhanced performances of the new UWB antenna. In order to do so, we have characterized the radiation of Trx antenna implementing a physical optics method that can analyze the field radiated by a lens [7]. When considering a resonant dipole, we assume a PWS current distribution varying with the frequency, whereas the aperture field for the enhanced leaky lens antenna is computed with the time domain solver of MWS CST with the slot radiating in an infinite dielectric medium (absorbing boundaries are used at the edges of the dielectric).

\section{RADIATED FIELDS}

As an Austin switch, we have considered a $240 \mu \mathrm{m}$ long dipole placed at the focal point of a $3 \mathrm{~mm}$ diameter silicon lens. The lens is a extended hemi-sphere designed at the elliptical synthesized position to maximum the gain. The fields radiated by such structure are presented in Fig. 1 in the uv-plane, and in Fig.2 in the e- and h-planes.

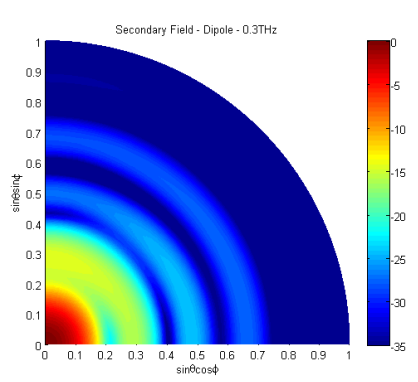

(a)

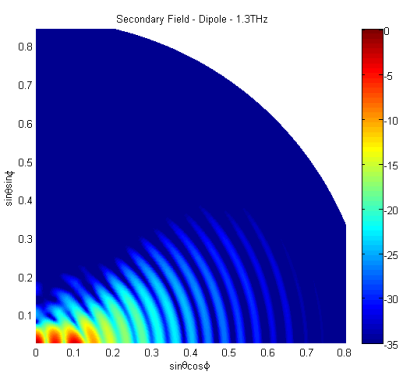

(b)
Fig.1 Radiated fields in the uv-plane for a $240 \mu \mathrm{m}$ long dipole coupled to a $3 \mathrm{~mm}$ diameter silicon lens at (a) $0.3 \mathrm{THz}$ and (b) $1.3 \mathrm{THz}$

One can clearly see that at high frequencies the field presents several lobes due to the large variation of the current distribution of a standard resonant dipole. The high variation of the field creates nulls at broadside that can alter the functionality of the TD system.

Next, we consider a enhanced leaky lens composed of a $50 \mu \mathrm{m}$ width infinite slot and a $25 \mu \mathrm{m}$ air cavity between the slot and the silicon. Such antenna is couple to a $3 \mathrm{~mm}$ silicon lens with slightly different extension chosen to maximize the directivity. The radiated fields are shown in Fig. 3 and 4. This antenna presents nearly constant patterns with no-nulls for a bandwidth larger than 1:10. 


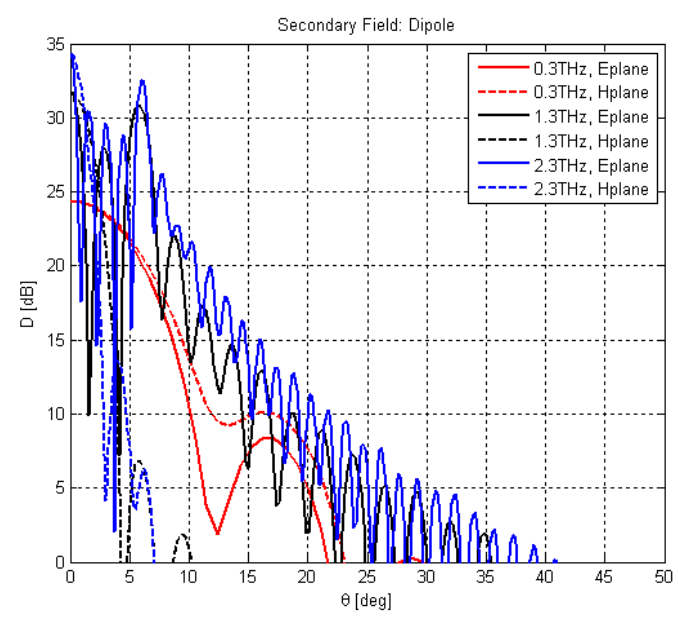

Fig.2 E-plane and H-plane radiated fields for the same antenna than Fig.1

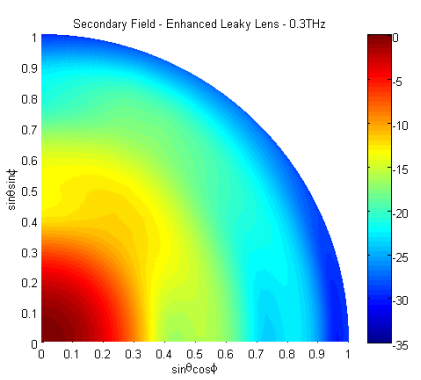

(a)

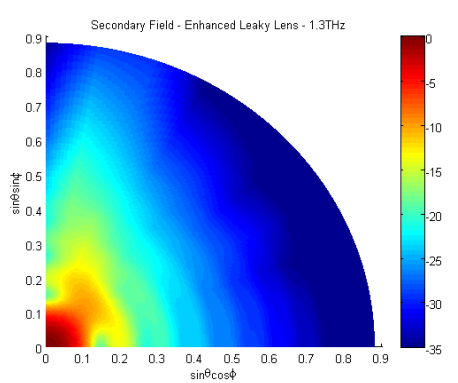

(b)
Fig.3 Radiated fields in the uv-plane for the enhanced leaky lens antenna at (a) $0.3 \mathrm{THz}$ and (b) $1.3 \mathrm{THz}$

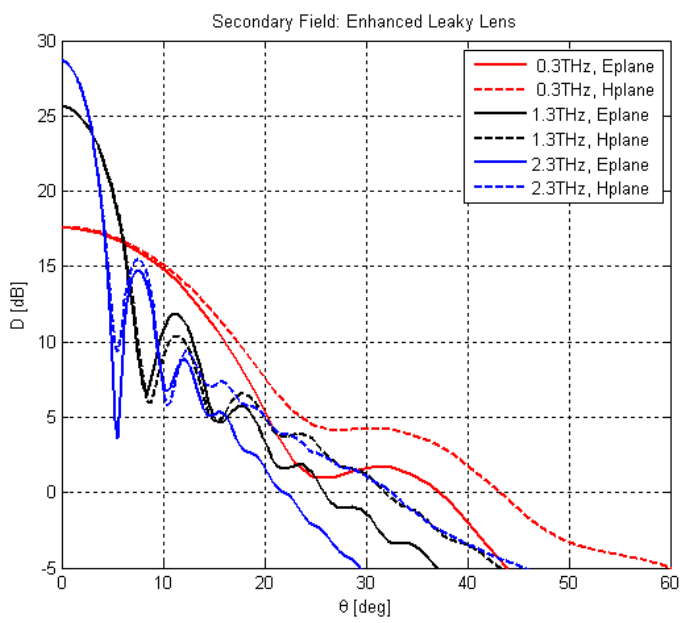

Fig.4 E-plane and H-plane radiated fields for the enhanced leaky lens antenna.

\section{GAIN VS. FREQUENCY}

Figure 6 presents the broadside gain as a function of the frequency for a 240um long dipole, and the UWB leaky lens. The gain is computed as the directivity minus the matching and reflection losses.

One can clearly observed the enormous advantage of UWB antenna. The resonant dipole presents several nulls at broadside making useful only the frequency range associated to the first lobe, whereas the UWB antenna has not null in such range. If one, integrates such gain over the useful bandwidth to obtained the total received power, the UWB leaky lens provides $15 \mathrm{~dB}$ more than the standard dipole. If we consider the same antennas also in reception, the improvement will be double.

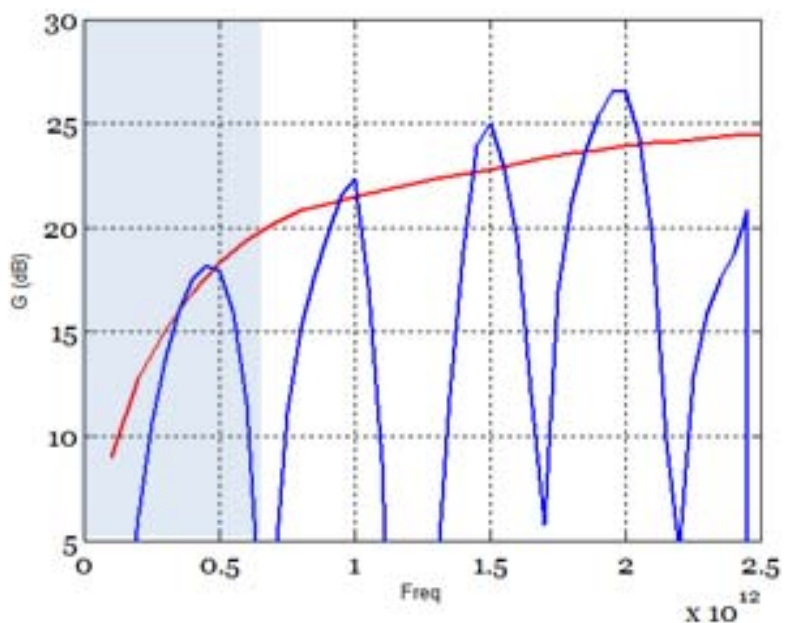

Fig.5 Gain as a function of the frequency: the blue line correspond to the resonant dipole and the red line to the enhanced leaky lens.

\section{CONCLUSIONS}

The enhanced leaky lens antenna has the potential to improve TD systems by over one order of magnitude. Key parameters of this antenna to achieve such performances are: nearly constant impedance, directive symmetric patterns and constant phase center over a decade of frequency bandwidth.

\section{REFERENCES}

[1] D. H. Auston, K. P. Cheung, and P. R. Smith, "Picosecond photoconducting Hertzian dipoles," Appl. Phys. Lett. Vol. 45, pp. 284286, Aug. 1984

[2] Smith, P.R.; Auston, D.H.; Nuss, M.C., "Subpicosecond photoconducting dipole antennas," Quantum Electronics, IEEE Journal of , vol.24, no.2, pp.255-260, Feb 1988

[3] A. Neto "UWB, Non Dispersive Radiation from the Planarly Fed Leaky Lens Antenna. Part 1: Theory and Design" IEEE Transactions of Antennas and Propagation, Vol. 58, No. 7 July 2010

[4] A. Neto, S. Monni, F. Nennie, "UWB, Non Dispersive Radiation from the Planarly Fed Leaky Lens Antenna. Part 2: Demonstrator and Measurements", IEEE Transactions of Antennas and Propagation, Vol. 58, No. 7 July 2010

[5] L. Duvillaret, F. Garet, J.-F. Roux, and J.-L. Coutaz, "Analytical Modeling and Optimization of Terahertz Time-Domain Spectroscopy Experiments Using Photoswitches as Antennas", IEEE Journal On Selected Topics In Quantum Electronics, vol. 7, no. 4, July/August 2001

[6] K. Ezdi, B. Heinen, C. Jördens, N. Vieweg, N. Krumbholz, R. Wilk, M. Mikulics, M. Koch, "A hybrid time-domain model for pulsed terahertz dipole antennas", Journal European Optical Society - Rapid Publications vol 4 09001, 2009

[7] D. F. Filippovic, S. S. Gearhart and G. M. Rebeiz, "Double Slot on Extended Hemispherical and Elliptical Silicon Dielectric Lenses", IEEE Trans. on MTT, Vol. 41, no. 10, Oct. 1993. 